\title{
Interacting host modifier systems control Wolbachia-induced cytoplasmic incompatibility in a haplodiploid mite
}

\author{
Nicky Wybouw ${ }^{1,2}$, Frederik Mortier ${ }^{1}$, and Dries Bonte ${ }^{1}$ \\ 1 Terrestrial Ecology Unit, Department of Biology, Faculty of Sciences, Ghent University, \\ Ghent, Belgium \\ 2 E-mail: nicky.wybouw@ugent.be
}

\begin{abstract}
Many reproductive parasites such as Wolbachia spread within host populations by inducing cytoplasmic incompatibility $(\mathrm{Cl})$. Cl occurs when parasite-modified sperm fertilizes uninfected eggs. In haplodiploid hosts, $\mathrm{Cl}$ can lead to different phenotypes depending on whether the fertilized eggs die or develop into males. Genetic conflict theories predict the evolution of host modulation of $\mathrm{Cl}$, which in turn strongly influences the stability of reproductive parasitism. Yet, despite the ubiquity of $\mathrm{Cl}$-inducing parasites in nature, there is no conclusive evidence for strong intraspecific host modulation of $\mathrm{Cl}$ strength and phenotype. Here, we tested for intraspecific host modulation of Wolbachia-induced $\mathrm{Cl}$ in haplodiploid Tetranychus spider mites. Using a single $\mathrm{Cl}$-inducing Wolbachia variant and mitochondrion, a Tetranychus urticae nuclear panel was created that consisted of infected and cured near-isogenic lines. We performed a highly replicated age-synchronized full diallel cross comprised of incompatible and compatible control crosses. We uncovered host modifier systems that strongly suppress $\mathrm{Cl}$ strength when carried by infected $T$. urticae males. Interspecific crosses showed that the male modifier systems suppress $\mathrm{Cl}$ strength across species boundaries. We also observed a continuum of $\mathrm{Cl}$ phenotypes in our crosses and identified strong intraspecific female modulation of $\mathrm{Cl}$ phenotype when paired with a specific male genotype. Crosses established a recessive genetic basis for the maternal effect and were consistent with polygenic Mendelian inheritance. Our findings identify spermatogenesis as an important target of selection for host suppression of $\mathrm{Cl}$ strength and underscore the importance of maternal genetic effects for the $\mathrm{Cl}$ phenotype. Both mechanisms interacted with the genotype of the mating partner, revealing that intraspecific host modulation of $\mathrm{Cl}$ strength and phenotype is underpinned by complex genetic architectures.
\end{abstract}

\section{Keywords}

host modification, reproductive parasitism, genetic conflict, pest control

\author{
Running title
}

Host modulation of parasite-induced $\mathrm{Cl}$ 


\section{Introduction}

Endosymbiotic reproductive parasites facilitate their maternal transmission by manipulating host reproduction [1,2]. Cytoplasmic incompatibility $(\mathrm{Cl})$ is the most common reproductive manipulation and induces defects in paternal chromosome condensation and segregation in developing embryos when infected males mate with uninfected females [3]. In diploid hosts, these defects in paternal chromosome behavior result in embryonic mortality, whereas in hosts with a haplodiploid mode of reproduction (unfertilized haploid eggs develop into males, fertilized diploid eggs result in females), two different $\mathrm{Cl}$ phenotypes can be distinguished. The chromosomal defects can give rise to viable haploid male offspring and is commonly referred to as Male Development $\mathrm{Cl}$ (MD-Cl) [4,5]. Alternatively, $\mathrm{Cl}$ in haplodiploids can lead to aneuploidy in fertilized eggs and induce female mortality in incompatible crosses (Female Mortality Cl, FM-Cl) [4-6]. Reproductive parasites can induce a mix of both $\mathrm{FM}-\mathrm{Cl}$ and $\mathrm{MD}-\mathrm{Cl}$ in haplodiploid arthropods, suggesting that the phenotypic outcomes of incompatible crosses can lie along a continuum with $\mathrm{FM}-\mathrm{Cl}$ and $\mathrm{MD}-\mathrm{Cl}$ as the extremes [4-9]. $\mathrm{Cl}$ is ablated when infected males mate with infected females and therefore provides a selective advantage to females that transmit the reproductive parasite.

$\mathrm{Cl}$ is primarily associated with Wolbachia, but other bacteria such as Rickettsiella, Cardinium, and Mesenet can also induce $\mathrm{Cl}$ in their arthropod host [3,10-13]. The genetic architecture of Wolbachia-mediated $\mathrm{Cl}$ is a pair of syntenic genes (cifA and cifB) that are located in WO prophage regions of certain Wolbachia genomes [14-16]. Across different diploid and haplodiploid host systems, $\mathrm{Cl}$ strength is highly variable and is typically associated with varying Wolbachia frequencies within host populations [17]. Although previous work has shown that natural genetic variation of cif operons can influence $\mathrm{Cl}$ strength [18-20], Wolbachia genetic diversity does not sufficiently explain $\mathrm{Cl}$ strength and phenotype variation. Interspecific transfer of Wolbachia and host species hybridization has revealed that host background can be a strong determining factor for $\mathrm{Cl}$ strength and phenotype [21-26]. For example, wMel Wolbachia induce weak $\mathrm{Cl}$ in its native host Drosophila melanogaster [23,24], but cause strong $\mathrm{Cl}$ in transinfected Drosophila simulans and Aedes aegypti $[25,26]$. In the haplodiploid wasp genus Nasonia, the Wolbachia strain determines Cl strength, whereas interspecific crosses uncovered a role for host genotype in the expression of $\mathrm{Cl}$ phenotype [8].

Interspecific modulation of $\mathrm{Cl}$ is consistent with genetic conflict theories that predict the evolution of host modifier systems in populations that are polymorphic for Wolbachia infection [27]. However, intraspecific modulation of $\mathrm{Cl}$ is not well documented, nor understood, in part due to variation caused by (asymmetrical) nuclear- or mitochondrial-associated incompatibilities [24,28-30]. Intraspecific variation in $\mathrm{Cl}$ strength as well as phenotype has been observed for the haplodiploid spider mite Tetranychus urticae and the parasitoid wasp Leptopilina heterotoma [4-6,31], raising questions about the ability of haplodiploid hosts to control the different features of $\mathrm{Cl}$. Despite the ubiquity of $\mathrm{Cl}$ in the natural world, the mechanistic underpinnings of host modulation are not known but can be characterized along several axes. Host modulation mechanisms can be expressed in males, females, and embryos of incompatible crosses. In males, these mechanisms limit Wolbachia-induced aberrations of spermatogenesis to produce normal, healthy sperm. Females can modify $\mathrm{Cl}$ through maternal effects that operate during oogenesis and embryogenesis. For instance, maternally supplied histones are vital for the formation of the male pronucleus and display a delayed deposition in embryos from incompatible crosses, findings that identify this process as a potential target for host selection [32,33]. Finally, the embryo can also modulate $\mathrm{Cl}$ throughout its development. Although $\mathrm{Cl}$ is commonly associated with defects in the early mitotic divisions [3], cytological assays have uncovered additional $\mathrm{Cl}$ phenotypes in later stages of embryonic development, such as regional mitotic failures [15]. Moreover, Wolbachia-induced $\mathrm{Cl}$ can also result in 
postembryonic mortality [7], dynamics that could allow for further selection for modifier systems in the offspring of incompatible crosses. Whether host modifier systems control different features of $\mathrm{Cl}$ or combine additively or synergistically is unknown, and are pertinent questions for our understanding of reproductive parasitism and the development of effective Cl-based pest management. Here, reproductive parasites are released into genetically diverse pest populations across the globe and reduce population growth by inducing strong $\mathrm{Cl}$. Segregating modifier systems in wild pest populations can severely threaten the long-term stability and effectiveness of $\mathrm{Cl}$-based pest control $[34,35]$.

To study the mechanistic underpinnings of host modulation in a haplodiploid arthropod, we created a nuclear panel of the spider mite $T$. urticae comprised of different nuclear backgrounds, a single mitochondrion, and a single $\mathrm{Cl}$-inducing Wolbachia variant. We performed a highly replicated full diallel cross and quantified variation in $\mathrm{Cl}$ strength and phenotype using Bayesian inference and corrected indexes that control for nuclear and temporal effects. Cl strength varied from very weak to complete and was strongly determined by the male genotype. Cl phenotypes ranged along a continuum and were determined by the female and male genotype as well as their interaction. Interspecific crosses were performed to study the control of male modifier systems when paired with a female genotype of a different Tetranychus species. Genetic crosses uncovered insights into the genetic architecture underlying intraspecific female modulation of the $\mathrm{Cl}$ phenotype. Together, our findings reveal multiple mechanisms of host modulation of $\mathrm{Cl}$ strength and phenotype within a single haplodiploid arthropod species.

\section{Materials and Methods}

\section{Creation and characterization of the Tetranychus nuclear panel}

A teleiochrysalid (virgin) female was collected from five Tetranychus field populations (Bch, Beis, Scp-w, Stt, and Temp) and from a laboratory population (LonX) that was derived from the London reference strain [36] (Table S1). To ensure near-isogenic nuclear backgrounds, lines were created by three sequential rounds of mother-son crosses. Genomic DNA was extracted from a pool of 20 adult females with a Quick-DNA Universal kit (BaseClear, the Netherlands). A fragment of the mitochondrial COI gene was sequenced for the Scp- $w$ and Bch lines (Sanger sequencing, MACROGEN Europe B.V.) (Table S2). Infection of the six Tetranychus lines with the reproductive manipulators Wolbachia, Rickettsia, Cardinium, and Spiroplasma was tested using diagnostic PCR assays. PCR assays were performed using DreamTaq DNA Polymerase (Life Technologies Europe B.V.) in a $50 \mu$ reaction mixture. PCR conditions are described in Table S2. A Wolbachia-infected Myrmica scabrinodis worker and laboratory populations of $T$. urticae that were infected with Wolbachia, Rickettsia, Cardinium, and Spiroplasma were used as positive controls. The diagnostic PCR assays showed that none of the lines carried Rickettsia, Cardinium, or Spiroplasma and that only Scp-w was infected with Wolbachia. The Wolbachia variant of Scp-w was further characterized by multilocus sequence typing (Sanger sequencing, MACROGEN Europe B.V.) (Table S2) [37]. We transferred the Wolbachia variant into four other near-isogenic backgrounds by paternal introgression, creating Beis- $w$, LonX- $w$, Stt- $w$, and Temp- $w$. For each near-isogenic nuclear background, 20 Wolbachia-infected Scp- $w$ females were crossed to 15 uninfected males, and 25 infected female offspring were backcrossed to 15 males of the uninfected genotype for an additional six generations (figure 1A). Introgressive transfer of Wolbachia into the Bch nuclear background failed since $F_{1}$ females did not oviposit. Hybrid sterility is a pervasive post-zygotic reproductive barrier between tetranychid species [38,39]. We morphologically classified the Bch line (electronic supplementary material). The fidelity of Wolbachia maternal transmission 
was tested in the five infected nuclear backgrounds of $T$. urticae. DNA was extracted from individual adult females and males (electronic supplementary material) and the diagnostic PCR assays were designed as described above (Table S2). The total numbers of tested mites are listed in Table S3. The Beis- $w$, LonX-w, Scp- $w$, Stt- $w$, and Temp-w lines were cured of Wolbachia infection by antibiotic treatment (electronic supplementary material). After antibiotic curing, the cured lines Beis-c, LonX-c, Scp-c, Stt-c, and Temp-c were maintained on detached bean leaves for at least four generations before Wolbachia-mediated incompatibilities were phenotyped. The 11 lines of the mite panel were maintained by serial passage on detached bean leaves at a census size of $\sim 250$ mites at $24^{\circ} \mathrm{C}, 60 \% \mathrm{RH}$, and a $16: 8$ light:dark photoperiod.

\section{Host modulation of Wolbachia-induced $\mathrm{Cl}$}

Host modifier systems were identified and characterized in our T. urticae panel by performing incompatible (uninfected females to infected males) and compatible (uninfected females to uninfected males) intraspecific crosses in a full factorial diallel cross design. This experimental design generated a total of 50 intraspecific cross types; 25 incompatible and 25 compatible control cross types. The control of $T$. urticae modifier systems in males of interspecific $\mathrm{Cl}$ was characterized by crossing $T$. urticae males of the five near-isogenic nuclear backgrounds to uninfected Bch females, generating an additional set of five incompatible and five compatible control cross types. To provide further validation that Bch represents a distinct species from $T$. urticae, five $F_{1}$ female teleiochrysalids were isolated per cross type (if present) and allowed to oviposit on $16 \mathrm{~cm}^{2}$ leaf discs until death. A third set of crosses tested the ability of infected females of Scp- $w$ and Beis- $w$ to rescue $\mathrm{Cl}$ and consisted of rescue (infected females to infected males) and control (infected females to uninfected males) crosses. Age cohorts of the mite lines were created by allowing 50 mated females to oviposit for 24 hours on detached bean leaves. Each cross type consisted of eight to eleven replicates except for the rescue and respective control crosses where five replicates were established per cross type (electronic supplementary material). For each replicate, five female teleiochrysalids were paired with four one- to three-day old adult males on a $16 \mathrm{~cm}^{2}$ leaf disc. After five days, mites were discarded and eggs were counted. During development, adult male and female offspring were isolated and counted.

\section{Genetic basis of female modulation of $\mathrm{Cl}$ phenotype}

We uncovered the mode of inheritance of female modulation of $\mathrm{Cl}$ phenotype using Scp-c and LonX-c females that express distinct $\mathrm{Cl}$ phenotypes when crossed to Scp- $w$ males (figure $2 A$ ). Heterozygous $F_{1}$ females were produced by reciprocal crosses between LonX-c and Scp-c. In each cross, 25 virgin females were paired with 20 adult males on a $16 \mathrm{~cm}^{2}$ leaf disc and allowed to mate and oviposit. During $F_{1}$ development, female teleiochrysalids were isolated for the incompatible and compatible control crosses. Adult Scp- $w$ and Scp-c males were obtained from synchronized age cohorts as previously described. Cross types consisted of seven to eight replicates, with five females and four males per replicate. After five days, mites were discarded and eggs were counted. During development, adult male and female offspring were isolated and counted.

To obtain recombinant $F_{2}$ females, 25 virgin $F_{1}$ females were backcrossed to 20 LonX$c$ males. Individual female $F_{2}$ teleiochrysalids were isolated, paired with a single one- to threeday old adult Scp-w or Scp-c male, and allowed to oviposit for seven days. Control cross types consisted of five to 21 replicates, whereas the incompatible cross types included 22 to 34 replicates. During development, adult male and female offspring were isolated and counted. 


\section{Statistical analysis of $\mathrm{Cl}$ strength and phenotype}

$\mathrm{Cl}$ strength and phenotype were analyzed with Bayesian inference using the brms package (version 2.12.0) in $R$ (version 3.6.3) [40,41]. Statistical models were run using Hamiltonian Monte Carlo (HMC) that implemented two chains with each 5,000 iterations from which 2,000 were warmup. We chose error distributions with 'biggest entropy'; the distribution with the least amount of assumptions that is consistent with the constraint of the outcome variable from first principles [42]. We modelled the proportion of adult female offspring over total number of eggs (F), proportion of adult male offspring over total number of eggs (MD), and the proportion of eggs that failed to generate adult mites over total number of eggs that did not generate adult males (FM) as responses with a binomial error distribution. These will be used to calculate informative $\mathrm{Cl}, \mathrm{MD}-\mathrm{Cl}$ and $\mathrm{FM}-\mathrm{Cl}$ metrics, respectively (see further). Priors were used that are weakly regularizing by choosing prior distributions that are significantly wider than the parameter values that would be reasonable to expect (electronic supplementary material). We evaluated the performance of every fitted model based on standardized procedures by checking mixing and stationarity in the trace plots and by checking the effective sample size and $\hat{R}$ statistic for each parameter [42].

We estimated F, MD, and FM using full models. For intraspecific crosses, F, MD, and FM were modelled with effects from the Wolbachia-infection state in $T$. urticae males, male genotype, female genotype, and all their interactions. For interspecific crosses, F, MD, and FM were modelled with effects from the Wolbachia-infection state in $T$. urticae males, male genotype, whether the cross was inter- or intraspecific, and all their interactions. Here, we assumed no effect of the T. urticae female genotype based on our model comparisons of the intraspecific crosses (see further). For all analyses above, the days at which the different cross types were initiated were included as variable intercepts. To study $\mathrm{Cl}$ rescue, we estimated $\mathrm{F}$ using the full model that incorporates all effects and their interactions. To study the inheritance of female modulation, F, MD, and FM were modelled with the effects from the Wolbachiainfection state in males and the female genotype. For $\mathrm{Cl}$ rescue and female modulation, the experimental design did not allow to estimate the variable day effect.

To control for variation in F, MD, and FM that is not related to Wolbachia-induced $\mathrm{Cl}$ (caused by nuclear and temporal effects), we used corrected indexes. Using the model estimates of $\mathrm{F}$, the corrected $\mathrm{Cl}$ strength $\left(\mathrm{Cl}_{\text {corr }}\right)$ for each cross type was calculated from the posterior distribution of the full generalized linear model:

$$
C I_{c o r r}=1-\frac{F_{o b s}}{F_{c}}
$$

where $F_{o b s}$ and $F_{c}$ are the estimated $F$ values in the incompatible and respective compatible crosses, respectively $[7,31]$. The corrected $M D-C I$ phenotype $\left(M_{\text {corr }}\right)$ was calculated using the estimated

$$
M D_{\text {corr }}=\frac{M D_{o b s}-M D_{c}}{1-M D_{c}}
$$
values;

where $M D_{\text {obs }}$ and $M D_{c}$ are the estimated $M D$ values in the incompatible and respective compatible crosses, respectively $[25,39,43]$. The corrected $\mathrm{FM}-\mathrm{Cl}$ phenotype $\left(\mathrm{FM}_{\text {corr }}\right)$ was calculated using the estimated FM values;

$$
F M_{\text {corr }}=\frac{F M_{o b s} *\left(1-M D_{c o r r}\right)-F M_{c}}{1-F M_{c}}
$$

where $\mathrm{FM}_{\mathrm{obs}}$ and $\mathrm{FM}_{\mathrm{c}}$ are the estimated $\mathrm{FM}$ values in the incompatible and respective compatible crosses, respectively (based on [25,39,43]). 
To understand the importance of male and female genotype and their interaction for intraspecific $\mathrm{Cl}$ strength and phenotype, we compared four variations of the full models that consistently assumed an effect of the Wolbachia-infection state in males but differed in the other fixed explanatory variables (male genotype, female genotype and their interactions) (electronic supplementary material). Models were compared using the Widely-Applicable information criterion (WAIC). To quantify the relative impact of the different explanatory variables, we compared the finite-population standard deviation of estimated coefficients for different explanatory variables and interactions in adjusted full models (electronic supplementary material). These adjusted full models estimated all effects as modelled variable effects instead of as fixed to improve our ability to interpret coefficients and their standard deviation accurately in a model with interactions. The standard deviation of the coefficients of the multiple model levels were visualized on the log-odds scale and compared to the standard deviation of the model residuals as a reference of unexplained variation.

Results

We created a $T$. urticae panel comprised of five near-isogenic nuclear backgrounds that shared a single mitochondrion and were either infected with a single $\mathrm{Cl}$-inducing Wolbachia variant (Beis-w, LonX-w, Scp- $w$, Stt- $w$, and Temp- $w$ ) or were cured of the infection (Beis-c, LonX-c, Scp-c, Stt-c, and Temp-c). A complete maternal transmission of Wolbachia was observed in all five infected $T$. urticae nuclear backgrounds (Table S3). All intraspecific compatible crosses produced $F_{1}$ females, demonstrating fertilization across all near-isogenic lines (electronic supplementary material). Morphological classification showed that the Bch line is distinct from $T$. urticae (electronic supplementary material) and compatible control crosses of $T$. urticae males of all near-isogenic lines and uninfected Bch females produced hybrid $F_{1}$ females. Virgin $F_{1}$ females did not oviposit across our cross types $(n=$ five females per cross type, if present), a post-zygotic reproductive barrier that is commonly observed in interspecific crosses with Tetranychus mites [38,39].

\section{Male modifier systems cause weak $\mathrm{Cl}$ in Tetranychus}

Using the model estimates of the full model (figure S1), we calculated the corrected $\mathrm{CI}$ strength $\left(\mathrm{Cl}_{\text {corr }}\right)$ across the intraspecific cross types, controlling for nuclear and temporal effects (figure $1 \mathrm{~B}$ ). Intraspecific $\mathrm{Cl}_{\text {corr }}$ varied greatly and ranged from complete to very weak. A model comparison was performed among models that differed in fixed explanatory variables to study the factors explaining intraspecific $\mathrm{Cl}$ strength variation (figure S2). These model comparisons revealed that the male genotype had the largest impact on model predictability, with its interaction with the female genotype as an important additional effect (figure S2). Variance analysis confirmed that the male genotype greatly determined intraspecific $\mathrm{Cl}$ strength with an additional substantial impact of the male-female genotype interaction (figure S3). In contrast, the coefficients of all other model levels exhibited markedly lower levels of variation (figure S3). These analyses indicate that (some) $T$. urticae male genotypes carry modifier systems that strongly modulate intraspecific $\mathrm{Cl}$ strength.

Wolbachia-infected Beis- $w$ males induced complete (or near-complete) $\mathrm{Cl}_{\text {corr }}$ when crossed to four uninfected female genotypes (Beis-c, Scp-c, Stt-c, and Temp-c). In contrast, all other genetic crosses revealed reduced levels of $\mathrm{Cl}_{\text {corr }}$. Weakest $\mathrm{Cl}_{\text {corr }}$ was observed in the crosses with infected Stt- $w$ and Temp- $w$ males, whereas infected Scp- $w$ and LonX- $w$ males induced intermediate levels of $\mathrm{Cl}_{\text {corr }}$ (figure 1B). The level of interaction of the male and female genotype on $\mathrm{Cl}_{\text {corr }}$ varied across the intraspecific crosses (figure $1 \mathrm{~B}$ and figure S4). The strongest interaction was observed when infected Beis- $w$ males were crossed to uninfected 
LonX-c females, resulting in a $\mathrm{Cl}_{\text {corr }}$ of $~ 75 \%$ (figure $1 \mathrm{~B}$ and figure S4). Together, these findings suggest that the LonX, Scp, Stt, and Temp genotypes carry nuclear modifier systems that are expressed in males and strongly reduce intraspecific $\mathrm{Cl}$ strength. The ability of infected females to rescue intraspecific $\mathrm{Cl}$ was confirmed for the Scp and Beis genotypes using replicated age-synchronized rescue (infected females to infected males) and control cross types (infected females to uninfected males) (figure S5).

The ability of Bch and T. urticae to hybridize allowed us to test the strength of the modifier systems in T. urticae males across species boundaries (figure $1 \mathrm{C}$ ). Here, we found that infected Beis- $w$ males continued to induce complete $\mathrm{Cl}_{\text {corr }}$, whereas crosses with the other $T$. urticae male genotypes resulted in lower levels of $\mathrm{Cl}_{\text {corr }}$ (figure 1C). Infected Temp-w males did not induce $\mathrm{Cl}_{\text {corr }}$ when crossed to uninfected Bch females, indicating a complete suppression of $\mathrm{Cl}$ by the host within this cross.

\section{Male modifier systems suppress $\mathrm{Cl}$ by controlling female mortality}

The total $\mathrm{Cl}$ strength in haplodiploids is the combined effect of $\mathrm{MD}-\mathrm{Cl}$ and $\mathrm{FM}-\mathrm{Cl}$ where the incompatibility is expressed as an excess of haploid male offspring or an increased mortality of female offspring, respectively. Using the model estimates of the full models, we calculated the corrected $\mathrm{MD}-\mathrm{Cl}$ and $\mathrm{FM}-\mathrm{Cl}$ indexes $\left(\mathrm{MD}_{\text {corr }}\right.$ and $\mathrm{FM}_{\text {corr, }}$, respectively) (figure S6 and figure S7). We observed a continuum for both $\mathrm{Cl}$ phenotypes within $T$. urticae, but $\mathrm{FM}_{\text {corr }}$ variation was considerably larger (figure $2 A$ ). The variation of $M D_{\text {corr }}$ and $F M_{\text {corr }}$ across the intraspecific crosses was examined by running several statistical models with the Wolbachiainfection state in males as a consistent explanatory variable. Model comparisons for MD-Cl indicated that male and female genotype and their interaction contributed equally to model predictability (figure S8) but accounted for a relatively low amount of variation (figure S9). In contrast, model comparisons and variance analysis uncovered that the male genotype and, to a lesser extent, its interaction with the female genotype were important determinants for FM$\mathrm{Cl}$, showing a correlation between $\mathrm{Cl}$ strength and $\mathrm{FM}-\mathrm{Cl}$ in our data (figure S8 and figure S9). Correlation plots confirmed that $\mathrm{FM}_{\text {corr }}$ and $\mathrm{Cl}_{\text {corr }}$ were tightly coupled, whereas patterns with $M D_{\text {corr }}$ were inconsistent (figure S10). Together, this suggests that the control of male modifier systems of intraspecific $\mathrm{Cl}$ strength was mainly regulated by changes in the mortality rate of female offspring (figure $2 \mathrm{~A}$ ).

Crossing LonX-w, Scp- $w$, and Stt- $w$ males to uninfected Bch females resulted in interspecific $\mathrm{Cl}$ that was mainly determined by $\mathrm{FM}_{\text {corr }}$ (figure $2 \mathrm{~B}$ ). In contrast, strong $\mathrm{MD}_{\text {corr }}$ was observed when Beis- $w$ males were crossed to Bch (figure $2 \mathrm{~B}$ ), revealing an interaction effect between the Beis and Bch genotypes for the expression of interspecific MD-Cl.

\section{A recessive maternal genetic effect contributes to intraspecific MD-Cl variation}

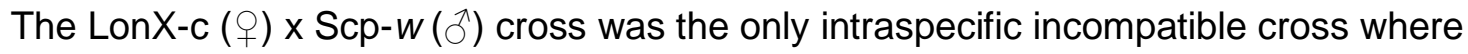
$\mathrm{MD}_{\text {corr }}(\sim 50 \%)$ exceeded $\mathrm{FM}_{\text {corr }}(\sim 12.5 \%)$ (figure $2 \mathrm{~A}$ and figure $\left.\mathrm{S} 10\right)$. Crossing Scp- $w$ males to other uninfected female genotypes caused no or very weak intraspecific $M D_{\text {corr }}$ (figure $2 A$ ), suggesting that the relatively strong $M D_{\text {corr }}$ resulted from an interaction between a maternal genetic feature of LonX with the Scp genotype. To gain further insight into female modulation of $\mathrm{Cl}$, we produced heterozygous $\mathrm{F}_{1}$ females by reciprocal crosses between LonX-c and Scp$\mathrm{C}$, and performed replicated incompatible and compatible crosses with Scp males (figure 2C). $\mathrm{Cl}$ strength was stable between parents and $\mathrm{F}_{1}$ offspring with the incompatible crosses of both sets of $\mathrm{F}_{1}$ females and Scp- $w$ males resulting in $\sim 70 \% \mathrm{Cl}_{\text {corr }}$ (figure $\mathrm{S} 11$ ). Crossing heterozygous $F_{1}$ females to $S c p-w$ males did not cause $M_{\text {corr }}$, establishing a recessive genetic basis for the LonX maternal effect (figure $2 \mathrm{C}$ ). We subsequently backcrossed $\mathrm{F}_{1}$ females to LonX-c males, and performed replicated incompatible and compatible crosses using a single 
recombinant $\mathrm{F}_{2}$ female per cross. $\mathrm{Cl}_{\text {corr }}$ remained stable and was $\sim 70 \%$ (figure $\mathrm{S} 11$ ). We observed $\mathrm{a} \sim 5 \%$ and $\sim 15 \% \mathrm{MD}_{\text {corr }}$ after crossing recombinant $\mathrm{F}_{2}$ females to Scp- $w$ (figure $2 \mathrm{C}$ ). We noted a clear change in the distribution of MD using recombinant $F_{2}$ females with LonX-c as the original maternal line (figure 2D). After mating with Scp-w males, 3 out of $34(8.8 \%)$ recombinant $F_{2}$ females produced a brood with an $M D$ value that exceeded the average $M D$ of LonX-c (figure 2D), patterns that are consistent with a polygenic basis.

\section{Discussion}

Intraspecific host modulation of parasite-induced $\mathrm{Cl}$ is predicted to influence the evolutionary trajectory of host-parasite interactions and, by suppressing $\mathrm{Cl}$ strength, the longterm effectiveness of $\mathrm{Cl}$-based pest management $[27,34,44]$. Here, we identified strong host modulation by the spider mite $T$. urticae of Wolbachia-mediated $\mathrm{Cl}$. We collected convincing evidence that a nuclear modifier system, or systems, segregates in $T$. urticae that strongly suppresses $\mathrm{Cl}$ strength when carried by Wolbachia-infected males. The strongest suppression of $\mathrm{Cl}$ was observed in infected Stt- $w$ and Temp- $w$ males (intraspecific $\mathrm{Cl}$ strength dropped as low as $\sim 15 \% \mathrm{Cl}_{\text {corr }}$ ). Crosses of $T$. urticae males to uninfected Bch females showed that the male modifier systems also strongly suppress $\mathrm{Cl}$ strength across species boundaries. Complete (or near-complete) $\mathrm{Cl}$ was only observed in the genetic crosses with infected Beis males, suggesting that only the Beis genotype lacks male suppressors within our T. urticae panel. Despite the ubiquity of $\mathrm{Cl}$-inducing parasites, evidence for intraspecific host suppression of $\mathrm{Cl}$ strength is rarely reported. Genetic work on Drosophila and Nasonia has gathered some evidence of modest intraspecific host modulation of $\mathrm{Cl}$ strength $[24,28,45]$. However, these results remain largely inconclusive due to a lack of control for (asymmetrical) nuclear- or mitochondrial-associated incompatibilities and line homozygosity. In $D$. simulans, Aedes aegypti, and Culex pipiens, no intraspecific genetic variation has (yet) been observed that modulates $\mathrm{Cl}$ strength despite intensive sampling efforts $[29,30,44,46,47]$. These findings are in sharp contrast with our study where we uncovered host suppression in four out of five isogenic lines. The maternal transmission of Wolbachia in tetranychid mites is sensitive to increased temperatures and is likely to be imperfect under natural conditions, preventing Wolbachia from reaching fixation and resulting in a persistent expression of $\mathrm{Cl}$ [48-50]. Our data therefore support models that predict the evolution of host modifiers in systems with strong $\mathrm{Cl}$ and imperfect maternal transmission $[2,27]$.

In contrast to $\mathrm{Cl}$, host nuclear suppressors that act against male-killing, a less common parasite-induced manipulation, have been described in a wide range of arthropod species, including fruit flies, butterflies, and spiders [2,51-53]. $\mathrm{Cl}$ and male-killing exert different selection pressures on the arthropod host [2]. Male-killing reduces the fitness of infected females that transmit the parasite, whereas $\mathrm{Cl}$ has deleterious effects for uninfected females. Moreover, in contrast to male-killing, the fitness cost of $\mathrm{Cl}$ is ablated in populations that are fixed for the reproductive parasite $[2,27]$. These divergent fitness penalties could explain the observed discrepancy in host modulation. Additionally, parasite-mediated male-killing of infected males is achieved by manipulating the dosage compensation system that regulates sex differentiation [54,55] and these pathways may be intrinsically more polymorphic and display greater standing genetic variation within host populations compared to those that are influenced by parasite-mediated $\mathrm{Cl}$.

The mechanism by which infected males are conditionally (partially) sterilized by Wolbachia is not well understood $[3,16,56]$, limiting our ability to unravel the mechanistic basis of host modulation. The host can develop toxicodynamic resistance to $\mathrm{Cl}$ by changes in the target-sites of Wolbachia Cif proteins (coined as the defensive model in [3]). As Wolbachia 
density has been observed to positively covary with $\mathrm{Cl}$ strength and maternal transmission efficiency [3,57], $\mathrm{Cl}$ can be overcome by genetic variants in the host that dysregulate Wolbachia density, a mechanism that can be viewed as toxicokinetic resistance (coined as the offensive model in [3]). In Nasonia wasps, host genetics of infected females contribute to variation in the maternal transmission of Wolbachia to the progeny [58]. In our study, maternal transmission of Wolbachia appeared complete in all infected genetic backgrounds, including the Stt and Temp genotypes that exhibited the strongest male suppression of $\mathrm{Cl}$. The interaction of the male modifier systems with the uninfected female genotype was an important determinant for $\mathrm{Cl}$ suppression, suggesting that variation in Wolbachia density in infected males does not (fully) explain $\mathrm{Cl}$ strength variation across our diallel cross design. In Wolbachia-infected wasps and fruit flies, $\mathrm{Cl}$ strength is coupled with Wolbachia cif gene expression [15,59], and host modulation of cif transcription could underlie the variation in $\mathrm{Cl}$ strength observed in our study. Cif genes are divided into a minimum of five phylogenetic clades and the encoded proteins exhibit extensive variation in domain structure [19]. Although all CifB proteins have a dimer of PD-(D/E)XK nuclease domains, the diversity of additional functional domains indicate that $\mathrm{Cl}$ may be manifested by different biochemical mechanisms across different Wolbachia variants, a hypothesis that finds some support in previous work $[3,16,19]$. Unfortunately, the cif repertoire of Wolbachia that infect $T$. urticae is not identified. Previous attempts to amplify and sequence cifA and cifB genes of Wolbachia in T. urticae using reference primers failed [43], indicating high gene sequence divergence from previously characterized cif genes. Multilocus sequence typing showed that the Cl-inducing Wolbachia variant of the current study belonged to supergroup $\mathrm{B}$, a clustering that is consistent with all previously characterized variants in $T$. urticae [43,60]. Wolbachia infection of Tetranychus mites is characterized by an apparent high strain diversity [60], raising the question of how the host modifier systems of our mite panel would interact with Wolbachia variants that carry divergent cif repertoires. Although speculative, the high prevalence of weak and intermediate $\mathrm{Cl}$ in $\mathrm{T}$. urticae populations across the globe could be (partially) caused by different male suppressors that segregate at high frequencies [4,43,61]. In Drosophila teissieri, genetic crosses suggest that $\mathrm{Cl}$ strength could be determined by an interaction between the Wolbachia variant and host genotype, but formal evidence awaits [28]. In D. melanogaster, suppressors of parasite-induced male-killing are located on different chromosomes and induce distinct suppressor phenotypes, indicative of independent origins of host suppression [52]. Further work is required to fully understand the mechanisms of male suppression of $\mathrm{Cl}$ in our T. urticae genotypes.

We also gathered evidence of strong intraspecific female modulation of $\mathrm{Cl}$ phenotype when paired with the Scp male genotype. In the wasp genus Nasonia, differences in $\mathrm{Cl}$ phenotype across species is also (partially) attributed to female modulation [8]. Consistent with the Nasonia system, the maternal genetic effect that contributes to MD-Cl in LonX is recessive [8]. Although the results of the backcross experiments appear consistent with polygenic Mendelian inheritance, the number of loci involved, their additivity and effect size remains unresolved. Multiple mechanisms can underpin female modulation of $\mathrm{MD}-\mathrm{Cl}$. During embryogenesis, the maternal genetic effect could contribute to the complete elimination of the paternal chromosomes, giving rise to viable haploid male offspring. Using high-copy library plasmids in yeast and a protein-interaction screen in Drosophila, Beckmann et al. collected evidence that shows that a particular type of Wolbachia CifB interacts with the maternally deposited proteins karyopherin- $\alpha$ and P32, and identified protamine-histone exchange and nuclear-protein import as target pathways [62]. As nuclear transport has previously been associated with genetic conflict $[63,64]$, it is tempting to speculate that these pathways underpin the observed maternal genetic effect of this study. Alternatively, the maternal genetic effect could result in (partial) fertilization failure by physiological changes within the female 
reproductive tissue. Future experiments that implement unbiased forward genetics are needed to uncover the genetic architecture of female modulation of $\mathrm{Cl}$ phenotype.

To conclude, we identified mechanisms of intraspecific host modulation that suppress $\mathrm{Cl}$ strength by male modifier systems and modify $\mathrm{Cl}$ phenotype by maternal genetic effects. As both mechanisms interacted with the genotype of the mating partner, we show that different complex genetic architectures underlie intraspecific host modulation of parasite-induced $\mathrm{Cl}$.

\section{Author Contributions}

NW conceived and designed experiments. NW and FM performed experiments. NW, FM, and DB analyzed data. NW wrote the manuscript with input from FM and DB.

\section{Acknowledgements}

We are indebted to Tadek and Helena Wybouw for their assistance during the field collections. We thank Philippe Auger for performing the morphological classification of the Tetranychus Bch line. NW was supported by a BOF post-doctoral fellowship (Ghent University, 01P03420) and by a Research Foundation - Flanders (FWO) Research Grant (1513719N). The authors declare no conflicts of interest.

\section{Data Accessibility}

The multilocus sequence type data of the $\mathrm{Cl}$-inducing Wolbachia variant are accessible at NCBI (OK669102- OK669106). The COI sequence data of the Bch and Scp-w line are accessible at NCBI (OL333561 and OL333562, respectively). Raw count data and scripts will be publicly available upon acceptance, or upon request.

\section{Figure Legends}

Figure 1. Host modifier systems suppress $\mathrm{Cl}$ strength in Tetranychus spider mites. (A) The experimental design that created the T. urticae nuclear panel. Wolbachia was transferred from Scp- $w$ into the Beis, LonX, Stt, and Temp nuclear backgrounds by paternal introgression, creating Beis- $w$, LonX- $w$, Stt- $w$, and Temp- $w$. Infected lines were cured of Wolbachia by antibiotic treatment, creating Beis-c, LonX-c, Scp-c, Stt-c, and Temp-c. The Wolbachia variant and mitochondrion of Scp- $w$ are indicated by encircled ' $w$ ' and ' $m$ ' symbols in red font. (B) Intraspecific $\mathrm{Cl}$ strength variation within the full diallel cross design. Crosses are ordered according to decreasing $\mathrm{Cl}$ strength. Violin plots are colour coded based on the male genotype (see bottom left). (C) Interspecific $\mathrm{Cl}$ strength variation using five $T$. urticae male genotypes and uninfected Bch females. Bch is a distinct Tetranychus species. Crosses are ordered according to decreasing intraspecific $\mathrm{Cl}$ strength. Violin plots are colour coded based on whether the cross was intraspecific or interspecific (see bottom left). For (B) and (C), Cl strength was estimated using the $\mathrm{Cl}_{\text {corr }}$ index that controls for variation caused by nuclear and temporal effects. Each violin plot represents the estimated average of $\mathrm{Cl}_{\text {corr }}$ for that cross and indicates the $0.09,0.50$ and 0.91 percentiles.

Figure 2. Host modulation of $\mathrm{Cl}$ phenotype in Tetranychus spider mites. (A) Intraspecific $\mathrm{Cl}$ phenotype variation within the full diallel cross design. Crosses are ordered according to decreasing $\mathrm{Cl}$ strength. (B) Interspecific $\mathrm{Cl}$ phenotype variation using five $T$. urticae male genotypes and uninfected Bch females. Bch is a distinct Tetranychus species. Crosses are ordered according to decreasing intraspecific $\mathrm{Cl}$ strength. (C) Inheritance of the maternal genetic effect that contributes to intraspecific $\mathrm{MD}-\mathrm{Cl}$ variation. For the heterozygous $\mathrm{F}_{1}$ and recombinant $F_{2}$ females, the genotype between brackets represents the original maternal 
genotype. All uninfected females were crossed to $S c p-w$ and Scp-c males. $M_{\text {corr }}$ and $F M_{\text {corr }}$ estimates of LonX-c and Scp-c are identical to those of panel A. (D) Distribution of MD in the incompatible and compatible crosses of recombinant $F_{2}$ females and Scp- $w$ and Scp-c males, respectively. Cross compatibility is colour coded (see middle right). The average MD values for LonX-c and Scp-c are shown in the bottom plot. For all panels, violin plots of $\mathrm{MD}_{\text {corr }}$ and $\mathrm{FM}_{\text {corr }}$ display a blue and red background, respectively (see middle right). Violin plots represent the estimated averages of $\mathrm{MD}_{\text {corr }}$ and $\mathrm{FM}_{\text {corr }}$ for each cross and indicate the 0.09, 0.50 and 0.91 percentiles.

\section{References}

1. Perlmutter JI, Bordenstein SR. 2020 Microorganisms in the reproductive tissues of arthropods. Nat Rev Microbiol 18, 97-111. (doi:10.1038/s41579-019-0309-z)

2. Engelstädter J, Hurst GDD. 2009 The Ecology and Evolution of Microbes that Manipulate Host Reproduction. Annu. Rev. Ecol. Evol. Syst. 40, 127-149.

(doi:10.1146/annurev.ecolsys.110308.120206)

3. Shropshire JD, Leigh B, Bordenstein SR. 2020 Symbiont-mediated cytoplasmic incompatibility: what have we learned in 50 years? eLife 9, e61989. (doi:10.7554/eLife.61989)

4. Perrot-Minnot M-J, Cheval B, Migeon A, Navajas M. 2002 Contrasting effects of Wolbachia on cytoplasmic incompatibility and fecundity in the haplodiploid mite Tetranychus urticae: Contrasting effects of Wolbachia in a haplodiploid mite. Journal of Evolutionary Biology 15, 808-817. (doi:10.1046/j.1420-9101.2002.00446.x)

5. Vavre F, Dedeine F, Quillon M, Fouillet P, Fleury F, Boulétreau M. 2001 Within-species diversity of Wolbachia-induced cytoplasmic incompatibility in haplodiploid insects. Evolution 55, 1710-1714. (doi:10.1111/j.0014-3820.2001.tb00691.x)

6. Breeuwer JAJ. 1997 Wolbachia and cytoplasmic incompatibility in the spider mites Tetranychus urticae and T. turkestani. Heredity 79, 41-47. (doi:10.1038/hdy.1997.121)

7. Nguyen DT, Morrow JL, Spooner-Hart RN, Riegler M. 2017 Independent cytoplasmic incompatibility induced by Cardinium and Wolbachia maintains endosymbiont coinfections in haplodiploid thrips populations. Evolution 71, 995-1008. (doi:10.1111/evo.13197)

8. Bordenstein SR, Uy JJ, Werren JH. 2003 Host genotype determines cytoplasmic incompatibility type in the haplodiploid genus Nasonia. Genetics 164, 223-233.

9. Vavre F, Fleury F, Varaldi J, Fouillet P, Bouleatreau M. 2000 Evidence for female mortality in Wolbachia -mediated cytoplasmic incompatibility in haplodiploid insects: Epidemiologic and evolutionary consequences. Evolution 54, 191-200. (doi:10.1111/j.00143820.2000.tb00019.x)

10. Hunter MS, Perlman SJ, Kelly SE. 2003 A bacterial symbiont in the Bacteroidetes induces cytoplasmic incompatibility in the parasitoid wasp Encarsia pergandiella. Proc. $R$. Soc. Lond. B 270, 2185-2190. (doi:10.1098/rspb.2003.2475)

11. Rosenwald LC, Sitvarin MI, White JA. 2020 Endosymbiotic Rickettsiella causes cytoplasmic incompatibility in a spider host. Proc. R. Soc. B. 287, 20201107. (doi:10.1098/rspb.2020.1107) 
12. Takano S, Gotoh Y, Hayashi T. 2021 “Candidatus Mesenet longicola”: Novel Endosymbionts of Brontispa longissima that Induce Cytoplasmic Incompatibility. Microb Ecol (doi:10.1007/s00248-021-01686-y)

13. Takano S et al. 2017 Unique clade of alphaproteobacterial endosymbionts induces complete cytoplasmic incompatibility in the coconut beetle. Proc Natl Acad Sci USA 114, 6110-6115. (doi:10.1073/pnas.1618094114)

14. Beckmann JF, Ronau JA, Hochstrasser M. 2017 A Wolbachia deubiquitylating enzyme induces cytoplasmic incompatibility. Nat Microbio/ 2, 17007.

15. LePage DP et al. 2017 Prophage WO genes recapitulate and enhance Wolbachiainduced cytoplasmic incompatibility. Nature 543, 243-247.

16. Beckmann JF, Bonneau M, Chen $H$, Hochstrasser M, Poinsot D, Merçot $H$, Weill $M$, Sicard M, Charlat S. 2019 The Toxin-Antidote Model of Cytoplasmic Incompatibility: Genetics and Evolutionary Implications. Trends in Genetics 35, 175-185. (doi:10.1016/j.tig.2018.12.004)

17. Hoffmann AA, Turelli M, Harshman LG. 1990 Factors affecting the distribution of cytoplasmic incompatibility in Drosophila simulans. 126, 933-948.

18. Beckmann JF, Van Vaerenberghe K, Akwa DE, Cooper BS. 2021 A single mutation weakens symbiont-induced reproductive manipulation through reductions in deubiquitylation efficiency. Proc Natl Acad Sci USA 118, e2113271118. (doi:10.1073/pnas.2113271118)

19. Martinez J, Klasson L, Welch JJ, Jiggins FM. 2020 Life and Death of Selfish Genes: Comparative Genomics Reveals the Dynamic Evolution of Cytoplasmic Incompatibility. Molecular Biology and Evolution , msaa209. (doi:10.1093/molbev/msaa209)

20. Shropshire JD, Rosenberg R, Bordenstein SR. 2021 The impacts of cytoplasmic incompatibility factor ( $\operatorname{cif} A$ and $\operatorname{cifB}$ ) genetic variation on phenotypes. Genetics 217, 1-13. (doi:10.1093/genetics/iyaa007)

21. Zabalou S et al. 2008 Multiple Rescue Factors Within a Wolbachia Strain. Genetics 178, 2145-2160. (doi:10.1534/genetics.107.086488)

22. Sakamoto H, Ishikawa Y, Sasaki T, Kikuyama S, Tatsuki S, Hoshizaki S. 2005 Transinfection reveals the crucial importance of Wolbachia genotypes in determining the type of reproductive alteration in the host. Genet. Res. 85, 205-210.

(doi:10.1017/S0016672305007573)

23. Holden PR, Jones P, Brookfield JFY. 1993 Evidence for a Wolbachia symbiont in Drosophila melanogaster. Genet. Res. 62, 23-29. (doi:10.1017/S0016672300031529)

24. Reynolds KT, Hoffmann AA. 2002 Male age, host effects and the weak expression or non-expression of cytoplasmic incompatibility in Drosophila strains infected by maternally transmitted Wolbachia. Genet. Res. 80, 79-87. (doi:10.1017/S0016672302005827)

25. Poinsot D, Bourtzis K, Markakis G, Savakis C, Merçot H. 1998 Wolbachia Transfer from Drosophila melanogaster into D. simulans: Host Effect and Cytoplasmic Incompatibility Relationships. Genetics 150, 227-237. (doi:10.1093/genetics/150.1.227)

26. Walker T et al. 2011 The wMel Wolbachia strain blocks dengue and invades caged Aedes aegypti populations. Nature 476, 450-453. (doi:10.1038/nature10355) 
27. Turelli M. 1994 Evolution of Incompatibility-inducing Microbes and Their Hosts. Evolution 48, 1500-1513. (doi:10.1111/j.1558-5646.1994.tb02192.x)

28. Cooper BS, Ginsberg PS, Turelli M, Matute DR. 2017 Wolbachia in the Drosophila yakuba Complex: Pervasive Frequency Variation and Weak Cytoplasmic Incompatibility, but No Apparent Effect on Reproductive Isolation. Genetics 205, 333-351. (doi:10.1534/genetics.116.196238)

29. Carrington LB, Lipkowitz JR, Hoffmann AA, Turelli M. 2011 A Re-Examination of Wolbachia-Induced Cytoplasmic Incompatibility in California Drosophila simulans. PLoS ONE 6, e22565. (doi:10.1371/journal.pone.0022565)

30. Hoffmann AA, Turelli M. 1988 Unidirectional incompatibility in Drosophila simulans: inheritance, geographic variation and fitness effects. Genetics 119, 435-444.

31. Mouton L, Henri H, Boulétreau M, Vavre F. 2005 Multiple infections and diversity of cytoplasmic incompatibility in a haplodiploid species. Heredity 94, 187-192. (doi:10.1038/sj.hdy.6800596)

32. Loppin B, Bonnefoy E, Anselme C, Laurençon A, Karr TL, Couble P. 2005 The histone H3.3 chaperone HIRA is essential for chromatin assembly in the male pronucleus. Nature 437, 1386-1390. (doi:10.1038/nature04059)

33. Landmann F, Orsi GA, Loppin B, Sullivan W. 2009 Wolbachia-Mediated Cytoplasmic Incompatibility Is Associated with Impaired Histone Deposition in the Male Pronucleus. PLoS Pathog 5, e1000343. (doi:10.1371/journal.ppat.1000343)

34. Ross PA, Turelli M, Hoffmann AA. 2019 Evolutionary Ecology of Wolbachia Releases for Disease Control. Annu. Rev. Genet. 53.

35. Utarini A et al. 2021 Efficacy of Wolbachia-Infected Mosquito Deployments for the Control of Dengue. N Engl J Med 384, 2177-2186. (doi:10.1056/NEJMoa2030243)

36. Grbić $M$ et al. 2011 The genome of Tetranychus urticae reveals herbivorous pest adaptations. Nature 479, 487-492. (doi:10.1038/nature10640)

37. Baldo L et al. 2006 Multilocus Sequence Typing System for the Endosymbiont Wolbachia pipientis. Applied and Environmental Microbiology 72, 7098-7110.

38. Dosse G, Langenscheidt M. 2009 Morphologische, biologische und histologische Untersuchungen an Hybriden aus dem Tetranychus urticea - cinnabarinus -Komplex (Acari, Tetranychidae)1. Zeitschrift für Angewandte Entomologie 54, 349-359. (doi:10.1111/j.1439-0418.1964.tb02950.x)

39. Cruz MA, Magalhães S, Sucena É, Zélé F. 2021 Wolbachia and host intrinsic reproductive barriers contribute additively to postmating isolation in spider mites. Evolution 75, 2085-2101. (doi:10.1111/evo.14286)

40. Bürkner P-C. 2018 Advanced Bayesian Multilevel Modeling with the R Package brms. The $R$ Journal 10, 395. (doi:10.32614/RJ-2018-017)

41. R Core Team. $2021 \mathrm{R}$ : A language and environment for statistical computing. $\mathrm{R}$ Foundation for Statistical Computing, Vienna, Austria. URL http://www.R-project.org.

42. McElreath R. 2018 Statistical Rethinking: A Bayesian Course with Examples in $R$ and Stan. 1st edn. Chapman and Hall/CRC. (doi:10.1201/9781315372495) 
43. Zélé F, Santos I, Matos M, Weill M, Vavre F, Magalhães S. 2020 Endosymbiont diversity in natural populations of Tetranychus mites is rapidly lost under laboratory conditions. Heredity 124, 603-617. (doi:10.1038/s41437-020-0297-9)

44. Ross PA et al. 2021 A w AlbB Wolbachia transinfection displays stable phenotypic effects across divergent Aedes aegypti mosquito backgrounds. Appl Environ Microbiol (doi:10.1128/AEM.01264-21)

45. Perrot-Minnot M-J, Werren JH. 1999 Wolbachia infection and incompatibility dynamics in experimental selection lines. , 272-282.

46. Atyame CM, Duron O, Tortosa P, Pasteur N, Fort P, Weill M. 2011 Multiple Wolbachia determinants control the evolution of cytoplasmic incompatibilities in Culex pipiens mosquito populations: Molecular Ecology 20, 286-298. (doi:10.1111/j.1365294X.2010.04937.x)

47. Duron O, Bernard C, Unal S, Berthomieu A, Berticat C, Weill M. 2006 Tracking factors modulating cytoplasmic incompatibilities in the mosquito Culex pipiens. Molecular Ecology 15, 3061-3071. (doi:10.1111/j.1365-294X.2006.02996.x)

48. Van Opijnen T, Breeuwer JAJ. 1999 High Temperatures Eliminate Wolbachia, a Cytoplasmic Incompatibility Inducing Endosymbiont, From the Two-spotted Spider Mite. Experimental and Applied Acarology 23, 871-881.

49. Breeuwer JAJ, Jacobs G. 1996 Wolbachia: intracellular manipulators of mite reproduction. Exp Appl Acarol 20, 421-434. (doi:10.1007/BF00053306)

50. Zélé F, Santos I, Olivieri I, Weill M, Duron O, Magalhães S. 2018 Endosymbiont diversity and prevalence in herbivorous spider mite populations in South-Western Europe. FEMS Microbiology Ecology 94. (doi:10.1093/femsec/fiy015)

51. Vanthournout B, Hendrickx F. 2016 Hidden suppression of sex ratio distortion suggests Red queen dynamics between Wolbachia and its dwarf spider host. J. Evol. Biol. 29, 1488-1494. (doi:10.1111/jeb.12861)

52. Kageyama D, Anbutsu H, Shimada M, Fukatsu T. 2009 Effects of host genotype against the expression of spiroplasma-induced male killing in Drosophila melanogaster. Heredity 102, 475-482. (doi:10.1038/hdy.2009.14)

53. Hornett EA, Charlat S, Duplouy AMR, Davies N, Roderick GK, Wedell N, Hurst GDD. 2006 Evolution of Male-Killer Suppression in a Natural Population. PLoS Biol 4, e283. (doi:10.1371/journal.pbio.0040283)

54. Veneti Z, Bentley JK, Koana T, Braig HR, Hurst GDD. 2005 A Functional Dosage Compensation Complex Required for Male Killing in Drosophila. Science 307, 1461-1463. (doi:10.1126/science.1107182)

55. Fukui T, Kawamoto M, Shoji K, Kiuchi T, Sugano S, Shimada T, Suzuki Y, Katsuma S. 2015 The Endosymbiotic Bacterium Wolbachia Selectively Kills Male Hosts by Targeting the Masculinizing Gene. PLoS Pathog 11, e1005048. (doi:10.1371/journal.ppat.1005048)

56. Hurst LD. 1991 The evolution of cytoplasmic incompatibility or when spite can be successful. Journal of Theoretical Biology 148, 269-277. 
57. Breeuwer JA, Werren JH. 1993 Cytoplasmic incompatibility and bacterial density in Nasonia vitripennis. Genetics 135, 565-574.

58. Funkhouser-Jones LJ, van Opstal EJ, Sharma A, Bordenstein SR. 2018 The Maternal Effect Gene Wds Controls Wolbachia Titer in Nasonia. Current Biology 28, 1692-1702.e6. (doi:10.1016/j.cub.2018.04.010)

59. Nasehi SF, Fathipour Y, Asgari S, Mehrabadi M. 2021 Environmental Temperature, but Not Male Age, Affects Wolbachia and Prophage WO Thereby Modulating Cytoplasmic Incompatibility in the Parasitoid Wasp, Habrobracon Hebetor. Microb Ecol (doi:10.1007/s00248-021-01768-x)

60. Zhang Y-K, Zhang K-J, Sun J-T, Yang X-M, Ge C, Hong X-Y. 2013 Diversity of Wolbachia in Natural Populations of Spider Mites (genus Tetranychus): Evidence for Complex Infection History and Disequilibrium Distribution. Microb Ecol 65, 731-739. (doi:10.1007/s00248-013-0198-z)

61. Gotoh T, Sugasawa J, Noda H, Kitashima Y. 2007 Wolbachia-induced cytoplasmic incompatibility in Japanese populations of Tetranychus urticae (Acari: Tetranychidae). Exp Appl Acarol 42, 1-16. (doi:10.1007/s10493-007-9072-3)

62. Beckmann JF, Sharma GD, Mendez L, Chen H, Hochstrasser M. 2019 The Wolbachia cytoplasmic incompatibility enzyme CidB targets nuclear import and protaminehistone exchange factors. eLife 8, e50026. (doi:10.7554/eLife.50026)

63. Tang S, Presgraves DC. 2009 Evolution of the Drosophila Nuclear Pore Complex Results in Multiple Hybrid Incompatibilities. Science 323, 779-782. (doi:10.1126/science.1169123)

64. Phadnis N, Hsieh E, Malik HS. 2012 Birth, Death, and Replacement of Karyopherins in Drosophila. Molecular Biology and Evolution 29, 1429-1440.

(doi:10.1093/molbev/msr306) 
A

Scp-w

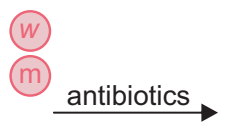

Scp-c

$-$ antibiotics
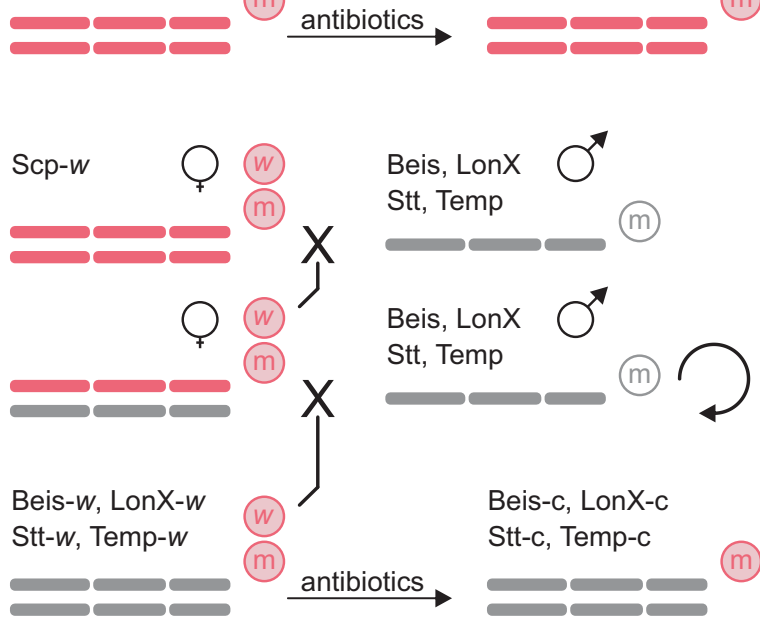

C

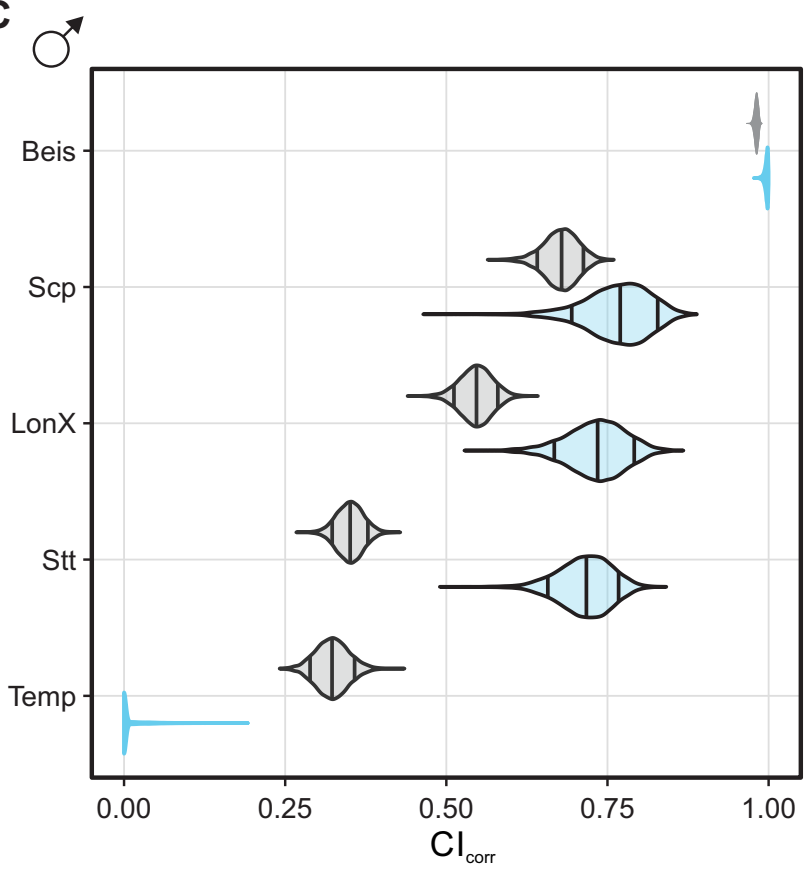

\section{(11)}

B

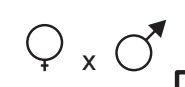

Temp-c $\times$ Beis

Scp-c $\times$ Beis

Beis-c $\times$ Beis

Stt-c $\times$ Beis

LonX-c x Beis

Temp-c x Scp

Stt-c $\times$ Scp

LonX-c x Scp

Beis-c x Scp

LonX-c x LonX

Scp-c x LonX

Beis-c $x$ LonX

Temp-c x LonX

Scp-c x Scp

Stt-c $\times$ Stt

Scp-c x Stt

Stt-c x LonX

Beis-c x Temp

Temp-c x Temp

LonX-c x Temp

Beis-c x Stt

Temp-c x Stt

Scp-c x Temp

Stt-c x Temp

LonX-c x Stt intraspecific

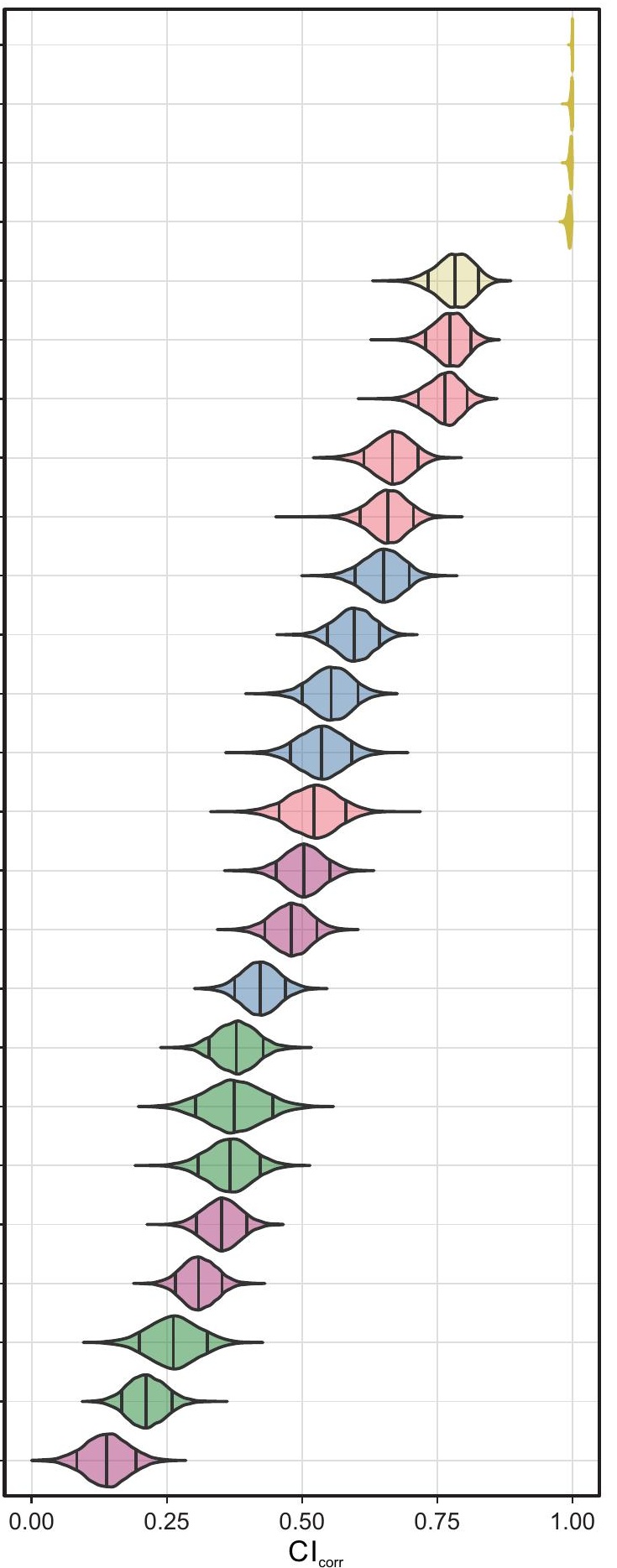


A $\bigodot_{x} \bigcirc^{*}$ Temp-c $\times$ Beis Scp-c $\times$ Beis

Beis-c $\times$ Beis

Stt-c $\times$ Beis

LonX-c x Beis

Temp-c x Scp

Stt-c x Scp

LonX-c x Scp -

Beis-c $x$ Scp

LonX-c x LonX -

Scp-c x LonX

Beis-c X LonX

Temp-c x LonX -

Scp-c x Scp

Stt-c $\times$ Stt

Scp-c x Stt

Stt-c $x$ LonX

Beis-c x Temp

Temp-c x Temp

LonX-c x Temp -

Beis-c $\times$ Stt

Temp-c x Stt

Scp-c x Temp -

Stt-c x Temp

LonX-c x Stt

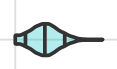

$\bowtie$
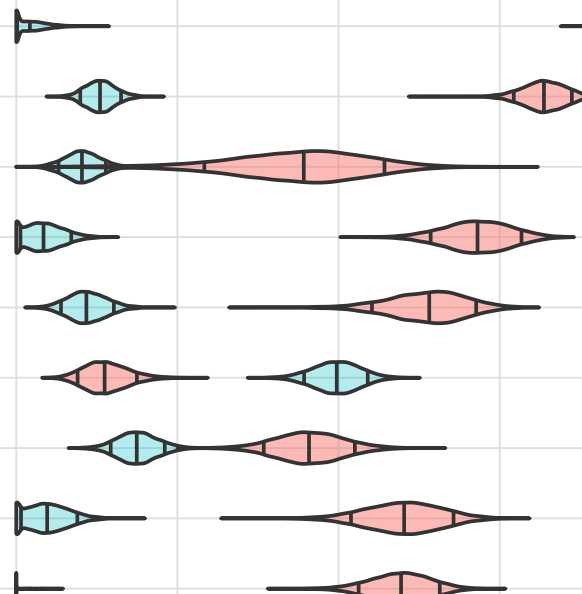

1

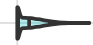
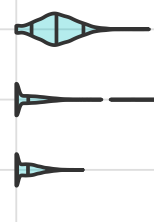

$p$

r

1
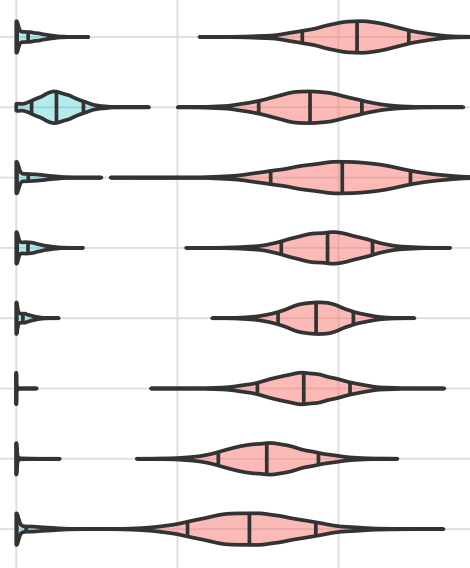

$1 D+1$

- D

$\infty \longrightarrow$

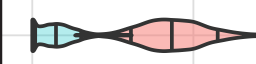

P
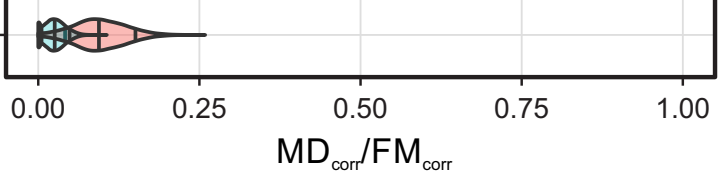

B
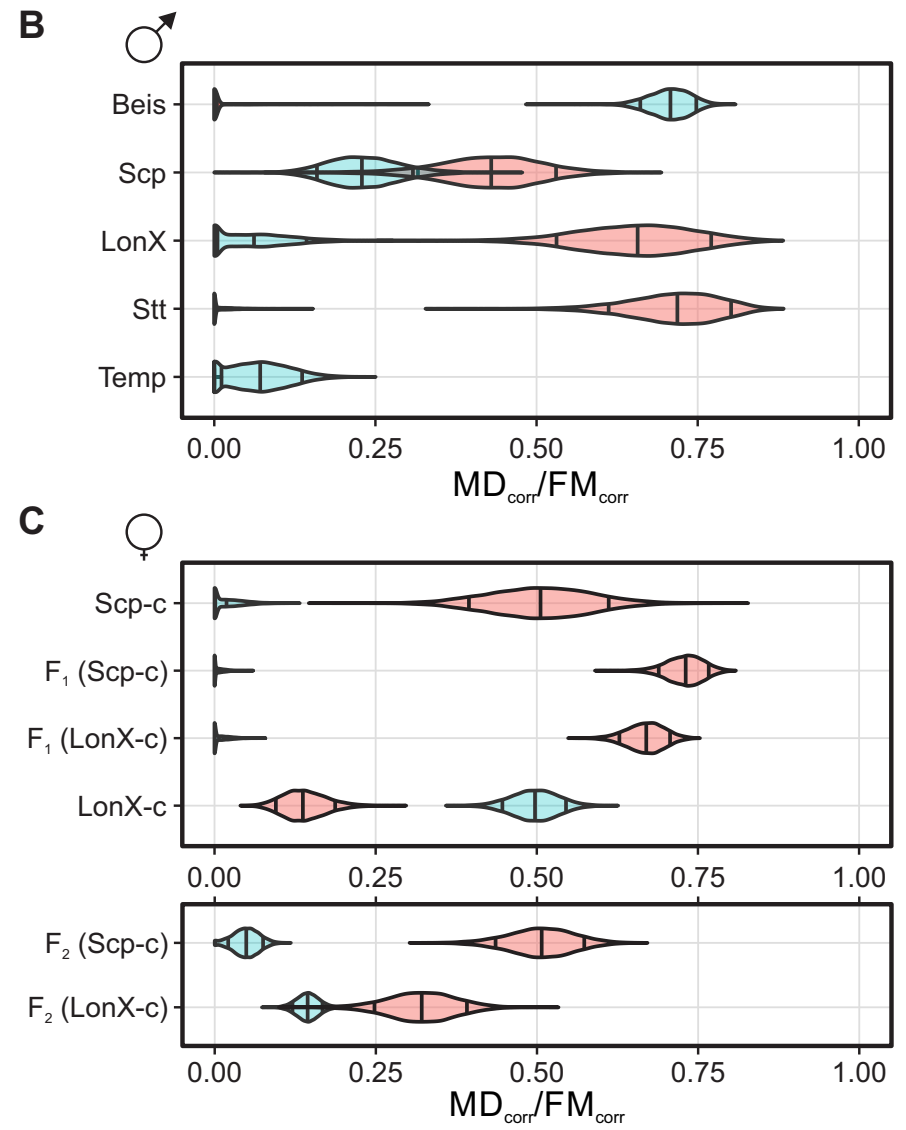

D

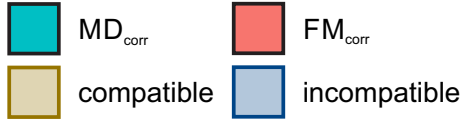

count

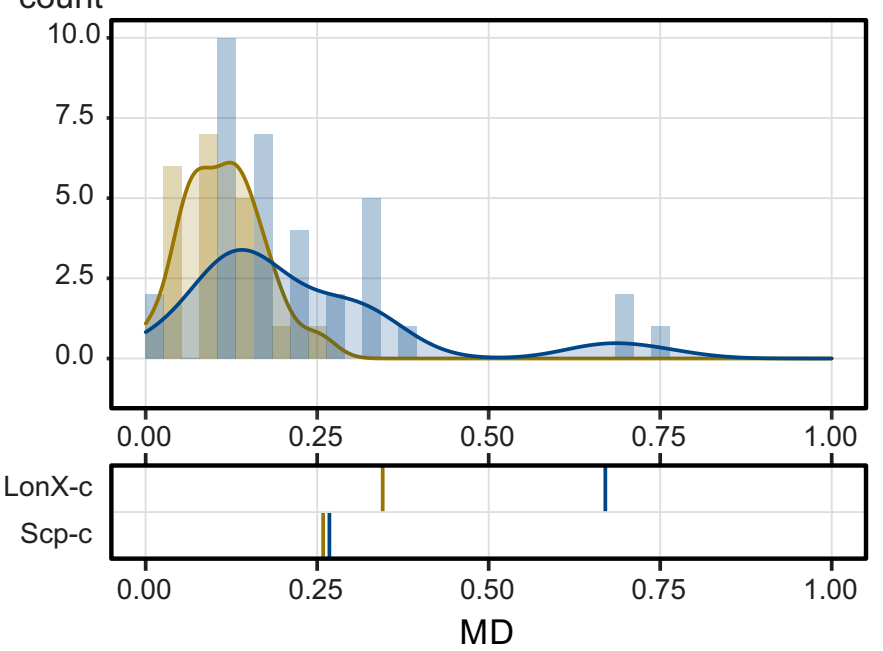

